\title{
Mannose-Binding Lectin is Associated with Thrombosis and Coagulopathy in Critically III COVID-19 Patients
}

\author{
Oskar Eriksson ${ }^{1, *}$ Michael Hultström ${ }^{2,3, *}$ Barbro Persson ${ }^{1} \quad$ Miklos Lipcsey ${ }^{2,4}$ \\ Kristina Nilsson Ekdahl ${ }^{1,5}$ Bo Nilsson ${ }^{1, * *}$ Robert Frithiof ${ }^{2, * *}$
}

${ }^{1}$ Department of Immunology, Genetics and Pathology, Uppsala University, Uppsala, Sweden

2 Department of Surgical Sciences, Anesthesiology and Intensive Care, Uppsala University, Uppsala, Sweden

${ }^{3}$ Department of Medical Cell Biology, Unit for Integrative Physiology, Uppsala University, Uppsala, Sweden

${ }^{4}$ Hedenstierna Laboratory, Department of Surgical Sciences,

Anesthesiology and Intensive Care, Uppsala University, Uppsala, Sweden

${ }^{5}$ Linnaeus Center for Biomaterials Chemistry, Linnaeus University,

Kalmar, Sweden

\author{
Address for correspondence Oskar Eriksson, MD, PhD, Klinisk \\ Immunologi, Rudbeck Laboratory, Dag Hammarskjölds väg 20, SE-751 \\ 85 Uppsala, Sweden (e-mail: oskar.eriksson@igp.uu.se). \\ Bo Nilsson, MD, PhD, Klinisk Immunologi, Rudbeck Laboratory, Dag \\ Hammarskjölds väg 20, SE-751 85 Uppsala, Sweden \\ (e-mail: bo.nilsson@igp.uu.se).
}

Thromb Haemost 2020;120:1720-1724.

\begin{abstract}
Keywords

- thrombosis

- COVID-19

- complement system

- mannose-binding lectin

The ongoing COVID-19 pandemic has caused significant morbidity and mortality worldwide, as well as profound effects on society. COVID-19 patients have an increased risk of thromboembolic (TE) complications, which develop despite pharmacological thromboprophylaxis. The mechanism behind COVID-19-associated coagulopathy remains unclear. Mannose-binding lectin (MBL), a pattern recognition molecule that initiates the lectin pathway of complement activation, has been suggested as a potential amplifier of blood coagulation during thromboinflammation. Here we describe data from a cohort of critically ill COVID-19 patients $(n=65)$ treated at a tertiary hospital center intensive care unit (ICU). A subset of patients had strongly elevated MBL plasma levels, and activity upon ICU admission, and patients who developed symptomatic TE (14\%) had significantly higher MBL levels than patients without TE. MBL was strongly correlated to plasma D-dimer levels, a marker of COVID-19 coagulopathy, but showed no relationship to degree of inflammation or other organ dysfunction. In conclusion, we have identified complement activation through the MBL pathway as a novel amplification mechanism that contributes to pathological thrombosis in critically ill COVID-19 patients. Pharmacological targeting of the MBL pathway could be a novel treatment option for thrombosis in COVID-19. Laboratory testing of MBL levels could be of value for identifying COVID-19 patients at risk for TE events.
\end{abstract}

The COVID-19 pandemic has spread rapidly around the world and caused significant morbidity and mortality, as well as profound economic and political impact on society. Thrombosis has emerged as a major COVID-19 complication despite routine

\footnotetext{
* Equal contribution as first authors.

** Equal contribution as last authors.
}

received

June 27,2020

accepted after revision

July 21,2020

thromboprophylaxis now being standard of care. ${ }^{1}$ COVID-19associated coagulopathy is characterized by strongly elevated plasma D-dimer levels and a hypercoagulable thromboelastography profile but normal or slightly elevated platelet counts. ${ }^{2}$ While the mechanisms behind the prothrombotic state in COVID-19 remain unclear, elevated markers of coagulation activation indicate a poor prognosis. ${ }^{3}$ The complement system,

(c) 2020. Thieme. All rights reserved. Georg Thieme Verlag KG,

Rüdigerstraße 14,

70469 Stuttgart, Germany
DOI https://doi.org/

10.1055/s-0040-1715835. ISSN 0340-6245. 
a plasma-based branch of the innate immune system, has been suggested to be involved in COVID-19 pathogenesis by exacerbating systemic inflammation and tissue damage and by amplifying the prothrombotic state. ${ }^{4}$ Specifically, mannosebinding lectin (MBL), a pattern recognition molecule that initiates the lectin pathway of complement activation, binds to coronaviruses ${ }^{5}$ and has been proposed to participate in the host defense during COVID-19 infection. ${ }^{6}$
To clarify the role of MBL in COVID-19 we measured plasma MBL levels and activity in a cohort of critically ill COVID-19 patients and investigated its relation to clinical outcome. A prospective single-center observational study was performed at the intensive care unit (ICU) of a tertiary hospital in Uppsala, Sweden (ClinicalTrials ID: NCT04316884). The study was approved by the Swedish National Ethical Review Agency (EPM; No. 2020-01623). Informed consent was obtained
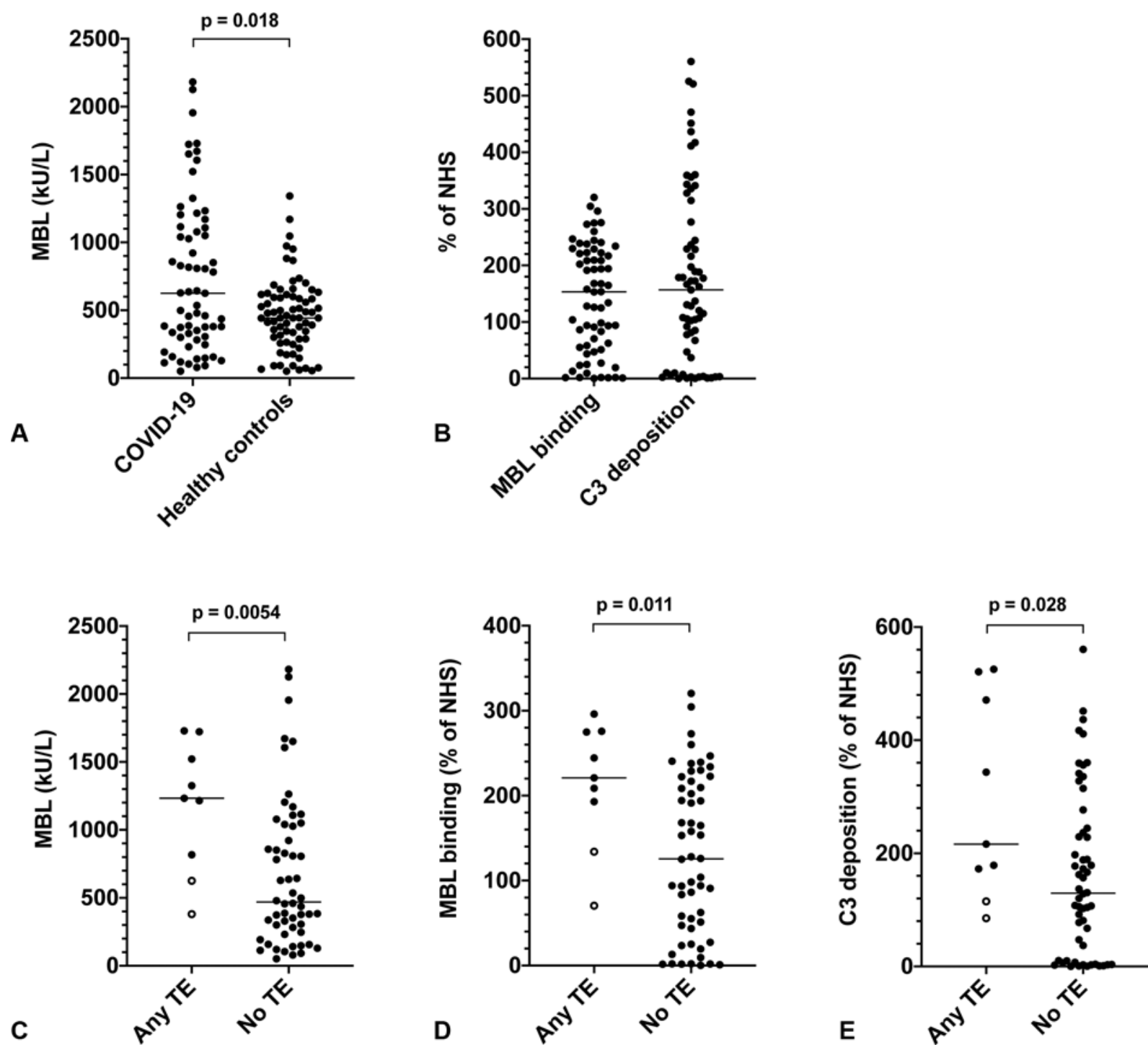

Fig. 1 Elevated mannose-binding lectin (MBL) levels and activity in critically ill COVID-19 patients are associated with thromboembolic events. (A) COVID-19 patients have elevated plasma MBL levels compared with healthy controls $(625 \mathrm{kU} / \mathrm{L}[303-1,112]$ in the patient group [ $n=65]$ vs. $444 \mathrm{kU} / \mathrm{L}$ [288-611] in controls [healthy blood donors, $n=72$ ], $p=0.018$ ). MBL was measured by an in-house sandwich enzyme-linked immunosorbent assay (ELISA) using a mouse monoclonal anti-MBL antibody (clone 3E7, Hycult Biotech) as capture antibody. The same antibody was biotinylated and used for detection together with streptavidin-horseradish peroxidase (HRP). (B) Elevated MBL pathway activity in COVID-19 patients measured as MBL binding activity (153\% [53-223]), and MBL-dependent C3 deposition (157\% [42-296]). MBL pathway activity was measured by a functional ELISA using mannan as MBL ligand. Microtiter plates were coated with $5 \mu \mathrm{g} / \mathrm{mL}$ mannan overnight and then incubated with plasma samples diluted in veronal-buffered saline at $37^{\circ} \mathrm{C}$ for 30 minutes. After washing, bound $\mathrm{MBL}$ and deposited $\mathrm{C} 3$ were detected by antibodies from R\&D Systems (AF2307) and Complement Technology (A213), respectively, and HRP-conjugated secondary antibodies. Results are expressed as percentage of the activity of pooled normal human serum (NHS). The MBL activity assay showed a very good correlation with the MBL antigen assay (Spearman's $r=0.94, p<0.0001$ ). (C-E) COVID-19 patients who develop thromboembolic complications have elevated plasma MBL levels and activity. Of the nine patients who developed thrombosis seven had pulmonary embolism (indicated by black dots) and two arterial thromboses (indicated by circles). (C) MBL plasma levels $(1,233 \mathrm{kU} / \mathrm{L}[721-1,623]$ in the thrombosis group vs. 470 kU/L [256-1,037] in patients with no thrombosis, $p=0.0054)$; (D) MBL activity (221\% [164-275] in the thrombosis group vs. 126\% [45-215] in patients with no thrombosis, $p=0.011$ ); (E) C3 deposition (216\% [144-496] in the thrombosis group vs. 129\% [10-243] in patients with no thrombosis, $p=0.028$ ). Results are expressed as medians and interquartile ranges (IQRs). $p$-Values were calculated using the Mann-Whitney $U$ test. 
from the patient, or next of kin if the patient was unable give consent. The Declaration of Helsinki and its subsequent revisions were followed. All patients $>18$ years of age with confirmed or suspected COVID-19 admitted to the ICU between March 13 and April 30, 2020, were screened for inclusion. Informed consents were given by 70 out of 71 screened patients. Two patients were excluded due to negative polymerase chain reaction for severe acute respiratory syndrome coronavirus 2. For two patients no initial blood sample was available. One patient was transferred from the ICU at another hospital and thus excluded. The remaining 65 patients were enrolled in the study.

Blood was sampled in ethylenediaminetetraacetic acid tubes, and plasma stored at $-70^{\circ} \mathrm{C}$ until analysis. Plasma MBL levels at day 1 at the ICU (on average COVID-19 day 10) were measured by an in-house enzyme-linked immunosorbent assay (ELISA), and were significantly higher than in healthy controls (-Fig. 1A). Activity of the MBL pathway was assessed by a functional ELISA using mannan as activator and MBL binding capacity and complement C3 deposition as readouts. ${ }^{7}$ This assay measures the functional MBL concentration in plasma and its capacity to activate complement, and confirmed elevated MBL activity and MBL-dependent C3 deposition in the patient group (-Fig. 1B).

Plasma MBL levels had no relation to survival (deceased $717 \mathrm{kU} / \mathrm{L}$ [379-1,139] [median and interquartile range] vs. survivors $499 \mathrm{kU} / \mathrm{L}$ [282-1,115], $p=0.62$ [Mann-Whitney $U$ test]), need for mechanical ventilation $(640 \mathrm{kU} / \mathrm{L}[302-1,156]$ vs. $460 \mathrm{kU} / \mathrm{L}$ [239-1,064], $p=0.54$ ), or acute kidney injury as measured by the KDIGO criteria $(p=0.55)$. In contrast, MBL was strongly related to thrombosis ( $\mathbf{F i g}$. 1C-E). A total of nine (14\%) patients developed a symptomatic thromboembolic (TE) event during their time at the ICU, identified by radiology performed on clinical indication. All patients received thromboprophylaxis with either dalteparin sodium (117 IE $/ \mathrm{kg}$ [91-152], $n=64$ ) or apixaban (5 mg, $n=1$ ). Patients with TE had significantly higher MBL levels compared with patients without TE, and MBL activity and MBLdependent $\mathrm{C} 3$ deposition showed the same relationship. Of the nine TEs, two were arterial thrombosis (stroke or myocardial infarction) and seven were pulmonary embolisms (PEs), in agreement with observations that PE is a frequent complication in COVID-19 patients. ${ }^{8,9}$ Interestingly, patients who developed PE all had MBL levels above the 95th percentile in controls (-Fig. 1C, D).

We next investigated the relationship between MBL levels and laboratory markers of coagulation activity, measured on the day of ICU admission ( - Table 1). MBL displayed a strong correlation with plasma D-dimer levels, corroborating the clinical association with TE. MBL was also significantly correlated to activated partial thromboplastin time, but not with prothrombin time or prothrombin fragment $1+2$. Interestingly, MBL appeared to specifically associate with biochemical markers of coagulation. No relationship

Table 1 Correlations between MBL and coagulation- and inflammation-related clinical chemistry laboratory tests

\begin{tabular}{|c|c|c|c|c|c|c|c|c|}
\hline \multirow[b]{2}{*}{ Analyte } & \multirow[b]{2}{*}{ Median (IQR) } & \multirow[b]{2}{*}{$n$} & \multicolumn{2}{|l|}{ MBL (kU/L) } & \multicolumn{2}{|c|}{ MBL activity (\% of NHS) } & \multicolumn{2}{|c|}{ C3 deposition (\% of NHS) } \\
\hline & & & Spearman's $r$ & $p$-Value & Spearman's $r$ & $p$-Value & Spearman's $r$ & $p$-Value \\
\hline $\begin{array}{l}\text { P-fibrin, D-dimer }(\mathrm{mg} / \mathrm{L}) \\
(<0.50)\end{array}$ & $1.50(0.90-2.70)$ & 61 & 0.39 & 0.0020 & 0.39 & 0.0018 & 0.31 & 0.015 \\
\hline APTT (s) (30-42) & $38(33-40)$ & 16 & -0.71 & 0.0030 & -0.76 & 0.0010 & -0.71 & 0.030 \\
\hline PT (INR) (0.9-1.2) & $1.1(1.0-1.2)$ & 19 & -0.28 & 0.25 & -0.38 & 0.11 & -0.46 & 0.049 \\
\hline $\begin{array}{l}\text { Prothrombin F 1+2 } \\
\text { (pmol/L) (69-229) }\end{array}$ & $672(555-822)$ & 51 & 0.11 & 0.45 & 0.16 & 0.27 & 0.053 & 0.71 \\
\hline $\begin{array}{l}\text { Platelet count }\left(\times 10^{9} / \mathrm{L}\right) \\
(150-350)\end{array}$ & $221(150-311)$ & 63 & 0.21 & 0.10 & 0.19 & 0.15 & 0.16 & 0.23 \\
\hline $\mathrm{P}-\mathrm{CRP}(\mathrm{mg} / \mathrm{L})(<5)$ & $169(118-237)$ & 63 & -0.075 & 0.56 & -0.035 & 0.79 & -0.041 & 0.75 \\
\hline P-interleukin-6 (ng/L) $(<7)$ & $103(46-184)$ & 37 & 0.062 & 0.72 & -0.046 & 0.79 & -0.091 & 0.59 \\
\hline P-ferritin $(\mu \mathrm{g} / \mathrm{L})(25-310)$ & $1,078(517-2,484)$ & 51 & 0.0022 & 0.99 & 0.038 & 0.79 & -0.14 & 0.33 \\
\hline $\mathrm{aB}-\mathrm{pO}_{2} / \mathrm{FiO}_{2}$ & $19(16-24)$ & 58 & -0.041 & 0.76 & -0.027 & 0.84 & 0.049 & 0.71 \\
\hline NT-proBNP (ng/L) $(<230)$ & $488(196-1,100)$ & 47 & 0.024 & 0.87 & 0.0021 & 0.99 & -0.069 & 0.64 \\
\hline C3 (g/L) (0.67-1.29) & $1.21(0.97-1.47)$ & 65 & 0.28 & 0.024 & 0.29 & 0.019 & 0.38 & 0.0019 \\
\hline C3d/C3 $($ ratio $* 1,000)(<5.3)$ & $5.5(4.4-7.7)$ & 65 & -0.21 & 0.088 & -0.19 & 0.12 & -0.29 & 0.018 \\
\hline C1q (mg/L) (70-300) & $90(65-110)$ & 65 & -0.072 & 0.57 & -0.028 & 0.82 & 0.000 & 0.99 \\
\hline
\end{tabular}

Abbreviations: aB- $\mathrm{pO}_{2} / \mathrm{FiO}_{2}$, arterial blood gas oxygen tension ratio to percentage of inspired oxygen; APPT, activated partial thromboplastin test; CRP, $\mathrm{C}-$ reactive protein; ELISA, enzyme-linked immunosorbent assay; F 1 + 2, fragment $1+2$; INR, international normalized ratio; IQR, interquartile range; MBL, mannose-binding lectin; NHS, normal human serum; NT-proBNP, N-terminal fragment of probrain natriuretic peptide; P-, plasma; PT prothrombin time. Note: Correlations were assessed by calculating Spearman's rank correlation coefficient (Spearman's $r$ ). Clinical chemistry test results were collected from the patients' clinical records, except C3, C3d, and C1q, which were measured by in-house methods, and prothrombin F $1+2$, which was measured by a commercial ELISA (Enzygnost F $1+2$, Siemens Healthcare).

Boldfaced values indicate statistically significant correlations ( $p$-Value $<0.05$ ). 
was found between MBL and markers of cardiac ( $\mathrm{N}$-terminal fragment of probrain natriuretic peptide) or respiratory function $\left(\mathrm{pO}_{2} / \mathrm{FiO}_{2}\right.$ ratio), nor with markers of inflammation (C-reactive protein, interleukin-6, or ferritin), in line with observations that MBL is not a typical acute phase reactant. ${ }^{10-12}$

MBL showed a significant correlation with total complement factor $\mathrm{C} 3$ levels, but not with the activation product C3d (measured as C3d/C3 ratio), a measure of activity of the alternative pathway of complement, nor with $\mathrm{C} 1 \mathrm{q}$, the initiator of the classical pathway. ${ }^{13}$ Total C3 levels, C3d/C3 ratio, or $\mathrm{C} 1 \mathrm{q}$ were not related to thrombotic events ( $p=0.75$, 0.57 , and 0.28 , respectively), indicating a specific association between MBL and thrombosis.

Our observations do not prove a causal role for MBL in thrombosis. Nonetheless, preclinical data implicate MBL as a key prothrombotic factor. MBL circulates in complex with the serine proteases mannose-associated serine protease (MASP)-1 and MASP-2, which activate the complement cascade upon MBL target binding. ${ }^{14}$ The MASPs intriguingly display coagulation factor-like substrate specificities ${ }^{15}$ and are activated during blood clotting. ${ }^{16}$ MASP-1 promotes clot formation by multiple mechanisms, including direct activation of factor XIII (FXIII) that cross-links fibrin. ${ }^{17}$ In addition, pharmacological or genetic targeting of the MBL pathway in mice provides protection from thrombosis, ${ }^{18,19}$ and MBL has been associated with thrombosis in epidemiological studies. ${ }^{20}$ The standard choice for thromboprophylaxis in COVID19 patients is low-molecular-weight heparin (LMWH), which preferentially targets FXa. Here, MBL-associated MASPs could act as a LMWH bypass mechanism to directly promote fibrin formation, and indeed, all the TE events in our study occurred despite thromboprophylaxis. As therapeutics targeting the MBL pathway are currently in clinical trials, ${ }^{21}$ this could be an alternative strategy for antithrombotic treatment in COVID-19.

A limitation of our study is that we did not assess the relationship between MBL and thrombosis in critically ill patients without COVID-19. Nonetheless, our data indicate a role for the MBL branch of the complement system in COVID19-associated coagulopathy and we propose that measurement of plasma MBL could be of value to identify COVID-19 patients at high risk of thrombosis.

\section{Authors' Contributions}

All authors participated in conception and design of the study. O.E. performed MBL activity assay, analyzed data, and prepared the manuscript. Thereafter, all authors had access to the data and participated in data collection and interpretation. All authors contributed to manuscript revision and gave approval of the final version.

\section{Funding}

The study was funded by grants from SciLifeLab/The Knut and Alice Wallenberg Foundation to M.H. (KAW2020.0182), the Swedish Research Council to R.F. (2014-02569 and 2014-07606), B.N. (2016-01060, 2020-05672), K.N.E. (2016-04519), and O.E. (2015-
06429), the Swedish Heart-Lung Foundation to B.N. (HLF 2020-0398), and by faculty grants from Linnaeus University to K.N.E.

\section{Conflict of Interest}

None declared.

\section{Acknowledgments}

The technical expertise of Sliva Abdalla is greatly appreciated. The authors thank the study nurses Elin Söderberg and Joanna Wessbergh, and the biobank research assistants Philip Karlsson and Erik Danielsson.

\section{References}

1 Al-Samkari H, Karp Leaf RS, Dzik WH, et al. COVID-19 and coagulation: bleeding and thrombotic manifestations of SARSCoV-2 infection. Blood 2020;136(04):489-500

2 Spiezia L, Boscolo A, Poletto F, et al. COVID-19-related severe hypercoagulability in patients admitted to intensive care unit for acute respiratory failure. Thromb Haemost 2020;120(06): 998-1000

3 Zhou F, Yu T, Du R, et al. Clinical course and risk factors for mortality of adult inpatients with COVID-19 in Wuhan, China: a retrospective cohort study. Lancet 2020;395(10229):1054-1062

4 Cugno M, Meroni PL, Gualtierotti R, et al. Complement activation in patients with COVID-19: a novel therapeutic target. J Allergy Clin Immunol 2020;146(01):215-217

5 Ip WKE, Chan KH, Law HKW, et al. Mannose-binding lectin in severe acute respiratory syndrome coronavirus infection. J Infect Dis 2005;191(10):1697-1704

6 Gao T, Hu M, Zhang X, et al. Highly pathogenic coronavirus N protein aggravates lung injury by MASP-2-mediated complement over-activation. medRxiv 2020;2020.03.29.20041962

7 Palarasah Y, Skjoedt M-O, Vitved L, Andersen TE, Skjoedt K, Koch C. Sodium polyanethole sulfonate as an inhibitor of activation of complement function in blood culture systems. J Clin Microbiol 2010;48(03):908-914

8 Klok FA, Kruip MJHA, van der Meer NJM, et al. Confirmation of the high cumulative incidence of thrombotic complications in critically ill ICU patients with COVID-19: an updated analysis. Thromb Res 2020;191:148-150

9 Cattaneo M, Bertinato EM, Birocchi S, et al. Pulmonary embolism or pulmonary thrombosis in COVID-19? Is the recommendation to use high-dose heparin for thromboprophylaxis justified? Thromb Haemost 2020;120(8):1230-1232

10 Dean MM, Minchinton RM, Heatley S, Eisen DP. Mannose binding lectin acute phase activity in patients with severe infection. J Clin Immunol 2005;25(04):346-352

11 Herpers BL, Endeman H, de Jong BA, et al. Acute-phase responsiveness of mannose-binding lectin in community-acquired pneumonia is highly dependent upon MBL2 genotypes. Clin Exp Immunol 2009;156(03):488-494

12 Perez-Castellano M, Peñaranda M, Payeras A, et al. Mannosebinding lectin does not act as an acute-phase reactant in adults with community-acquired pneumococcal pneumonia. Clin Exp Immunol 2006;145(02):228-234

13 Ekdahl KN, Persson B, Mohlin C, Sandholm K, Skattum L, Nilsson B. Interpretation of serological complement biomarkers in disease. Front Immunol 2018;9:2237

14 Eriksson O, Mohlin C, Nilsson B, Ekdahl KN. The human platelet as an innate immune cell: interactions between activated platelets and the complement system. Front Immunol 2019;10:1590

15 Garred P, Genster N, Pilely K, et al. A journey through the lectin pathway of complement-MBL and beyond. Immunol Rev 2016; 274(01):74-97 
16 Kozarcanin H, Lood C, Munthe-Fog L, et al. The lectin complement pathway serine proteases (MASPs) represent a possible crossroad between the coagulation and complement systems in thromboinflammation. J Thromb Haemost 2016;14(03):531-545

17 Hess K, Ajjan R, Phoenix F, Dobó J, Gál P, Schroeder V. Effects of MASP-1 of the complement system on activation of coagulation factors and plasma clot formation. PLoS One 2012;7(04):e35690

18 Pavlov VI, Tan YS, McClure EE, et al. Human mannose-binding lectin inhibitor prevents myocardial injury and arterial thrombogenesis in a novel animal model. Am J Pathol 2015;185(02):347-355
19 Orsini F, Villa P, Parrella S, et al. Targeting mannose-binding lectin confers long-lasting protection with a surprisingly wide therapeutic window in cerebral ischemia. Circulation 2012;126(12): 1484-1494

20 Liang RA, Høiland II, Ueland T, et al. Plasma levels of mannosebinding lectin and future risk of venous thromboembolism. J Thromb Haemost 2019;17(10):1661-1669

21 Mastellos DC, Ricklin D, Lambris JD. Clinical promise of nextgeneration complement therapeutics. Nat Rev Drug Discov 2019; 18(09):707-729 